\title{
Are Employees in Oil and Gas Companies More Vulnerable to Hypertension Than the General Population of Working Age? Evidence From Men and Women in Northwest of China
}

\section{Ying Liang}

Fourth Military Medical University: Air Force Medical University

Tianle Che

State Key Laboratory of Pathogen and Biosecurity,Beijing Institute of Microbiology and Epidemiology,Beijing,China

Kyriacos Kyriacou

Department of Economics and Finance,Brunel University,UK

Siming Liu

Department of Economics and Finance,Brunel University,UK

Haiyue Zhang

Fourth Military Medical University: Air Force Medical University

Ling Li

Administrative Affairs Centre, Changqing Oil Field Filiale,Xi'an,Shaanxi Province,China

Hai Mi

Administrative Affair Centre,Changqing Oil Field Filiale,Xi'an, Shaanxi Province,China

Lei Shang

Fourth Military Medical University: Air Force Medical University

Zhijun Tan ( $\nabla$ tanzjfmmu@126.com )

Fourth Military Medical University: Air Force Medical University https://orcid.org/0000-0003-0902-7122

\section{Research}

Keywords: oil and gas companies, hypertension, general population, working age, employees, men and women, Northwest of China

Posted Date: May 26th, 2021

DOI: https://doi.org/10.21203/rs.3.rs-541908/v1

License: (c) (1) This work is licensed under a Creative Commons Attribution 4.0 International License. Read Full License 



\title{
Are employees in oil and gas companies more vulnerable to hypertension than the general population of working age? Evidence from men and women in Northwest of China
}

Ying Liang ${ }^{1 \#}$, Tianle Che ${ }^{2 \#}$, Kyriacos Kyriacou ${ }^{3}$, Siming Liu ${ }^{3}$, Haiyue Zhang ${ }^{1}$, Ling $\mathrm{Li}^{4}$, Hai $\mathrm{Mi}^{4}$, Shang Lei ${ }^{1}$, Zhijun Tan ${ }^{1}$

1 Department of Health Statistics, Fourth Military Medical University, Xi'an, Shaanxi Province, China; Ministry of Education Key Lab of Hazard Assessment and Control in Special Operational Environment, The Airforce Military Medical University, Xi'an,710032 Shaanxi Province, China

2 State Key Laboratory of Pathogen and Biosecurity, Beijing Institute of Microbiology and Epidemiology, Beijing, China

3 Department of Economics and Finance, Brunel University, UK

4 Administrative Affairs Centre, Changqing Oil Field Filiale, Xi'an, Shaanxi Province, China

\begin{abstract}
Objective To examine whether the employees of an oil and gas company in Shaanxi Province are more vulnerable to hypertension than the general residents, aged 18-60, living in the same geographical region.

Design Cross-sectional analysis of the hypertension prevalence of two different population by using propensity score matching (PSM).

Participants Employees of Changqing Oil Field Filiale took part in Health Risk Factors Survey (2013) and residents took part in the National Health Service Survey (2013) in Shaanxi Province, China.

Main outcome measures The primary outcome was hypertention which was defined as systolic blood pressure of at least $140 \mathrm{~mm} \mathrm{Hg}$, or diastolic blood pressure of at least $90 \mathrm{~mm} \mathrm{Hg}$, or self-reported antihypertensive medication use in the previous 2 weeks, or self-reported history of hypertention.
\end{abstract}

Results Changqing employees were much younger, with a higher proportion of men, and their lifestyle was less healthy, with higher BMI, more drinkers and smokers. Before PSM, hypertension prevalence of Changqing employees was slightly lower than Shaanxi residents (5.8\% vs 7.6\%). After PSM, the results of the adjusted logistic model showed that Changqing employees were more likely to be hypertensive $(O R=1.10$, 95\% $C I=1.02-1.19, P=0.01)$. Among the male samples, Changqing employees were more likely to be hypertensive $(O R=1.43,95 \% C I=1.31-1.58, P<0.01)$, while the opposite was true for the female samples $(\mathrm{OR}=0.64,95 \% \mathrm{CI}=0.56-0.73, \mathrm{P}<0.01)$.

1 *Correspondence Author

Email Adresses for the Correspondence Author:

Zhijun Tan: tanzjfmmu@126.com 
Conclusion Changqing's employees, as a whole, and male employees in particular, were more vulnerable to hypertension than the general residents of the region. However, the reverse was found for female employees.

\section{Introduction}

Hypertension is an important health challenge around the world primarily due to its high prevalence but also because it is one of the leading risk factors of cardiovascular disease, premature death and disability. ${ }^{1-3}$ For low- and middle-income countries, like China, the prevention and control of hypertension are essential, as the prevalence of hypertension in these countries has been increasing since $2000 .{ }^{4-6}$ In order to effectively prevent and control hypertension, more health resources should be invested in the working population (employees) because the majority of employees are adults aged 1860 and this age range is the key period for hypertension prevention. ${ }^{7}$

For employees in oil and gas industries, health risk factors include not only unhealthy lifestyles but also occupational hazards coming from exposure to various industrial pollutions. ${ }^{8}$ Employees in petrochemical industries are regularly engaged in long work shifts (12 hours) that often occur at night and some live in remote regions far away from their families. These working conditions are often associated with high calorific intake, fatty diets with poor fiber, physical inactivity, worse sleep quality, and high level of job strain. ${ }^{8-9}$ Some studies have shown that the unhealthy lifestyles listed above can contribute to increased cardiovascular disease risk and this, in turn, may also affect productivity. ${ }^{10-12}$ Employees in oil and gas industries are more likely to be exposed to gas flaring, oil-polluted surfaces and underground water than the general population. ${ }^{13-14}$ Evidence suggests that chronic high-level and prolonged low-level exposure to these contaminants increases cardiovascular risk including hypertension. ${ }^{15-}$ 18

In China most oil and gas companies are large state-owned enterprises. Compared to the private sector labor force, employees in state-owned companies have unique advantages in coping with the damage caused by chronic diseases. For example, they have access to a better health care system, jointly established by companies and the government. This provides almost free medical treatment, free periodic physical examinations and a wider scope of health management services. ${ }^{19}$ In addition, the income of employees in oil and gas companies are much higher on average, which allows them to better respond to health risks.

Following on from the previous discussion this study asks, are the employees of oil and gas industries more vulnerable to hypertension than the general population of 
China? We also ask, do the protective factors (e.g. better health care and higher incomes) effectively reduce the prevalence of hypertension? Furthermore, some studies have shown that there are significant gender differences of the exposures to risk factors of hypertension. ${ }^{20-22}$ For example, women in oil and gas companies are more likely to work in offices or logistics, and their occupational exposure is lower than that of men. Therefore, an additional question that can be asked is whether a gender difference exists in the vulnerability to hypertension? The answers to the questions raised above are essential for the health management of oil and gas industry. More generally, they are also directly relevant to health resource allocation and policy making.

In order to address these questions, this study uses the employees of Changqing Oil Field Company, one of the largest oil and gas companies in China, and residents aged 18-60 in Shaanxi Province, where the company is located, to conduct a quantitative comparative study. However, it is difficult to compare the prevalence of hypertension between the two samples directly because many hypertension-associated risk factors (for example age, gender, and health behavior) are distributed differently between the two groups. Therefore, before comparing the prevalence of hypertension, the distributions of possible hypertension-associated factors should be balanced between the two groups. The Propensity Score Matching (PSM) method, ${ }^{23}$ which is widely used in observational studies to balance covariate distributions between study groups, is used in this study. The primary aim of this current study is to ascertain whether the employees of an oil and gas company in Shaanxi Province are more vulnerable to hypertension than the general residents aged 18-60 living in Shaanxi Province. Also, we examine whether a gender difference exists in the vulnerability.

\section{Methods}

\section{Health Risk Factors Survey (HRFS) in Changqing Oil Field Filiale 24}

Changqing Oil Field has organized annual health examinations for employees since 2007. In 2013, in addition to the annual health examination, a Health Risk Factors Survey was performed. The survey questionnaire was developed by the Fourth Military Medical University. The content of the questionnaire consisted of 11 sections and more than 30 questions, including demographic factors, physical characteristics, personal disease history, family disease history, smoking, diet, physical activity, sleep, mental condition, and living environment. The survey was organized by the company's health management department, and was conducted in the form of a face-to-face interview. The interviewers were recruited from health administrators at all levels of the company 
and were trained before the survey was implemented. All the 25 secondary units of the company participated in the survey and 52,000 questionnaires were distributed. In total, 50,013 valid questionnaires were collected. The validity of the survey questionnaires was checked by health administrators before data entry.

\section{National Health Service Survey (NHSS) in Shaanxi Province, China}

In Shaanxi's 2013 NHSS, a representative sample including 32 districts/counties, 160 sub-districts/townships, 320 residential committees/villages and 20,702 households was randomly selected by using a 3-stage cluster random sampling method. A total of 57,529 participants finished the interview. Instructions for performing face-

to-face interviews on NHSS questions were provided by the National Health and Family Planning Commission (NHFPC) of China. The interviewers were recruited from local health workers and trained by supervisors recruited from local health authority staff who were trained by the NHFPC. Shaanxi adopted the NHSS form with the same questionnaire, survey and quality control methods, and conducted the survey at the same time as the national survey. If participants were able to answer the questions in writing, they personally filled out the forms, following the instructions provided. If the participants were unable to write, a trained interviewer would collect the necessary information from respondents in face-to-face interviews. The questionnaire used for the NHSS in 2013 included more than 200 questions involving diseases, hospitalizations, health-related behaviors, socioeconomic factors, vaccinations, disease control and health services specific to women and children.

\section{Statistical Analysis Methods}

\section{Definition of the study sample}

The employees in Changqing Oil Field were adult, in-service individuals, most between 19 to 60 years old. However, the participants of the NHSS cover a wider age range. In order to improve comparability, only 19-60 year old participants were selected in this study. The analysis was conducted using samples containing 49,698 Changqing employees and 34,820 residents from Shaanxi province (after excluding cases with missing values in key analysis variables).

\section{Definition of hypertension}

For the employees of Changqing, we defined hypertension as systolic blood pressure of at least $140 \mathrm{~mm} \mathrm{Hg}$, or diastolic blood pressure of at least $90 \mathrm{~mm} \mathrm{Hg}$, or self-reported antihypertensive medication use in the previous 2 weeks. For the residents 
of Shaanxi, chronic conditions including hypertension were self-reported and the interviewers ensured that the chronic conditions were clinically diagnosed by medical organizations to minimize any mistakes due to self-reporting.

\section{Definition of health risk factors}

The sociodemographic factors selected from the two surveys in this study included gender, age, body mass index (BMI), height and weight. Age was divided into three categories, 19-30, 31-45, 46-60. BMI was also divided into three categories including normal or underweight $\left(<24 \mathrm{Kg} / \mathrm{M}^{2}\right.$ ), overweight $\left(24-28 \mathrm{Kg} / \mathrm{M}^{2}\right)$, obese $\left(>28 \mathrm{Kg} / \mathrm{M}^{2}\right)$. Health behavior included smoking (no smoking, smoking cessation and smoking) and drinking (drinking more than one time a week and no drinking).

\section{Statistical analysis}

The propensity score was calculated by the logistic regression model, in which Changqing employees were taken as the treatment group and the residents of Shaanxi Province as the control group. Before PSM, z-tests were used to compare the means difference of continuous variables and Chi-Square tests were used to compare the proportions difference of categorical variables. P-values smaller than 0.05 were statistically significant. Health risk factors including gender, drinking and smoking as categorical variables along with age and BMI as continuous variables, were incorporated in the logistic model as covariates. The nearest neighbor matching method was used with a matching ratio of $1: 1$ and a caliper value of 0.038 . This resulted in 26,178 matched cases. Absolute standardized difference in mean (ASDM) was adopted to evaluate the matching results and a confounding factor was considered to be balanced between treatment and control group when ASDM was smaller than 0.1. ${ }^{25}$ After PSM, two types of logistic models were used to test whether Changqing staff were more vulnerable to hypertension than Shaanxi residents. In the unadjusted logistic model, group of population (Changqin or Shaanxi) was the only covariate, while in the adjusted logistic model, those baseline variables with P-values smaller than 0.1 in the balance tests were added as covariates to adjust the bias further. The PSM was conducted by using the SPSS's plug-in "psmatching 3.02", and the remaining statistical analysis and drawing were done by SPSS 24 and R 3.2.5.

\section{Patients and public involvement}

Patients were not involved in this study. 


\section{Results}

\section{The characteristics of the samples before PSM}

The characteristics of demographic factors and other health risk factors of the samples before PSM are shown in Table 1. Compared with the control group, the proportions of male participants and the younger age groups are much higher in the treatment group (male: $62.7 \%$ vs 49.7\%; 19-30 years: 30.4\% vs 19.9\%; 31-45 years: $53.1 \%$ vs $36.2 \%)$. However, health behaviors were much unhealthier in the treatment group (overweight or obesity: $36.8 \%$ vs $24.9 \%$; smoking: $34.8 \%$ vs 33\%; drinking more than once a week: $32.4 \%$ vs $23.1 \%$ ). All comparisons are statistically significant $(\mathrm{P}<0.01)$.

Table 1 The basic characteristics of the samples before PSM

\begin{tabular}{|c|c|c|c|}
\hline Variables & Treatment group & Control group & $P$ Value \\
\hline Male $(n, \%)$ & $31,176(62.7)$ & $\begin{array}{r}17,289 \\
(49.7)\end{array}$ & $<0.01$ \\
\hline Age $($ Mean \pm SD $)$ & $36.3 \pm 8.8$ & $42.3 \pm 11.3$ & $<0.01$ \\
\hline \multicolumn{4}{|l|}{ Age group } \\
\hline $19-30(\mathrm{n}, \%)$ & $15,119(30.4)$ & $6,934(19.9)$ & $<0.01$ \\
\hline $31-45(\mathrm{n}, \%)$ & $26,389(53.1)$ & $12,613(36.2)$ & \\
\hline $46-60(\mathrm{n}, \%)$ & $8,190(16.5)$ & $15,273(43.9)$ & \\
\hline Height $($ Mean \pm SD $)$ & $168.7 \pm 13.2$ & $165.4 \pm 9.5$ & $<0.01$ \\
\hline Weight $($ Mean \pm SD $)$ & $66.5 \pm 7.6$ & $61.1 \pm 7.0$ & $<0.01$ \\
\hline BMI $($ Mean \pm SD $)$ & $23.3 \pm 3.8$ & $22.3 \pm 2.9$ & $<0.01$ \\
\hline Normal or underweight (n, \%) & $31,402(63.2)$ & $\begin{array}{r}26,181 \\
(75.2)\end{array}$ & $<0.01$ \\
\hline Overweight (n, \%) & $14,425(29.0)$ & $7,471(21.5)$ & \\
\hline Obese $(\mathrm{n}, \%)$ & $3,871(7.8)$ & $1,168(3.4)$ & \\
\hline $\begin{array}{l}\text { Drinking more than once a } \\
\text { week }(\mathbf{n}, \%)\end{array}$ & $15,560(32.4)$ & $7,000(23.1)$ & $<0.01$ \\
\hline \multicolumn{4}{|l|}{ Smoking $(\mathbf{n}, \%)^{*}$} \\
\hline Non-smoking & $32,399(65.2)$ & $\begin{array}{r}23,332 \\
(67.0)\end{array}$ & $<0.01$ \\
\hline Smoking cessation & $1,476(3.0)$ & $703(2.0)$ & \\
\hline Smoking & $15,823(31.8)$ & $\begin{array}{r}10,785 \\
(31.0)\end{array}$ & \\
\hline
\end{tabular}

\section{The prevalence of hypertension before PSM}

The prevalence of hypertension of the overall sample and different strata, before PSM, is shown in Table 2. The overall hypertension prevalence of the treatment group 
was much lower than the control group $(5.8 \%$ vs $7.6 \%, \mathrm{P}<0.01)$. As the stratified results show, some stratum's prevalence was much lower in the treatment group - such as those who were female, non-drinking, non-smoking and those in any BMI stratum - while the prevalence in other strata was much lower in the control group. All comparisons are statistically significant $(\mathrm{P}<0.01)$.

Table 2 The prevalence of hypertension in each stratum before PSM (N*,\%)

\begin{tabular}{llll}
\hline Factors & Treatment group & Control group & P Value \\
\hline Gender & & & \\
$\quad$ Male & $2,449(7.9)$ & $1,154(6.7)$ & $<0.01$ \\
Female & $456(2.5)$ & $1,482(8.5)$ & $<0.01$ \\
Age & & & \\
$\quad 19-30$ & $184(1.2)$ & $15(0.2)$ & $<0.01$ \\
$31-45$ & $1,369(5.2)$ & $428(3.4)$ & $<0.01$ \\
$46-60$ & $1,352(16.5)$ & $2,193(14.4)$ & $<0.01$ \\
BMI & & & \\
Normal or underweight & $845(2.7)$ & $1,372(5.2)$ & $<0.01$ \\
Overweight & $1,456(10.0)$ & $1,004(13.4)$ & $<0.01$ \\
Obese & $604(16.2)$ & $260(22.3)$ & $<0.01$ \\
Drinking & & & \\
Drinking & $1,574(10.1)$ & $440(6.3)$ & $<0.01$ \\
Non-drinking & $1,331(3.9)$ & $2,196(7.9)$ & $<0.01$ \\
Smoking & & & \\
Non-smoking & $1,124(3.5)$ & $1,835(7.9)$ & $<0.01$ \\
Smoking cessation & $277(18.8)$ & $97(13.8)$ & $<0.01$ \\
Smoking & $1,504(9.5)$ & $704(6.5)$ & $<0.01$ \\
Overall & $2,905(5.8)$ & $2,636(7.6)$ & $<0.01$ \\
\hline
\end{tabular}

*N was the number of subjects who were hypertensive.

\section{Evaluation of the balance of covariates after PSM}

After PSM, the distributions of the baseline factors between treatment and control groups are shown in Table 3. Most of the baseline factors were balanced between the two groups $(\mathrm{P}>0.1)$ except for weight, $\mathrm{BMI}$ (both continuous and categorical variables) and age (both continuous and categorical variables).

Table 3 The distributions of the baseline factors after PSM

\begin{tabular}{llll}
\hline \multicolumn{1}{c}{ Variables } & Treatment group & Control group & $\boldsymbol{P}$ value \\
\hline Male $(\mathbf{n}, \boldsymbol{\%})$ & $14,026(53.6)$ & $13,959(53.3)$ & 0.56 \\
Height $($ Mean \pm SD) & $166.6(7.4)$ & $166.5(6.8)$ & 0.14 \\
Weight $($ Mean \pm SD) & $62.8(10.9)$ & $62.5(9.9)$ & $\mathbf{0 . 0 1}$ \\
BMI $($ Mean \pm SD) & $22.5(3.3)$ & $22.5(3.0)$ & 0.08 \\
$\quad$ Normal or underweight & $2,017(7.7)$ & $1,777(6.8)$ & $<\mathbf{0 . 0 1}$ \\
$\quad$ Overweight & $16,791(64.1)$ & $17,207(65.7)$ &
\end{tabular}




\begin{tabular}{clll} 
Obesity & $7,370(28.2)$ & $7,194(27.5)$ & \\
Age $($ Mean \pm SD) & $39.0(9.0)$ & $38.8(10.6)$ & $\mathbf{0 . 0 2}$ \\
$\quad 19-30$ & $5,268(20.1)$ & $6,933(26.5)$ & $<\mathbf{0 . 0 1}$ \\
$31-45$ & $14,459(55.2)$ & $11,725(44.8)$ & \\
$46-60$ & $6,451(24.6)$ & $7,520(28.7)$ & \\
Drinking & $6,228(23.8)$ & $6,254(23.9)$ & 0.79 \\
Smoking & & & \\
$\quad$ No smoking & $17,678(67.5)$ & $17,612(67.3)$ & 0.77 \\
smoking cessation & $601(2.3)$ & $618(2.4)$ & \\
Smoking & $7,899(30.2)$ & $7,948(30.4)$ & \\
\hline
\end{tabular}

As shown in Table 4 and Figue 1, the ASDMs of most covariate variables were larger than 0.1 before PSM, except for status of smoking. After PSM, all of them became smaller than 0.1 , which indicated that all the covariate variables were balanced between the two groups.

Table 4 The SDMs of the covariates before and after PSM

\begin{tabular}{lcc}
\hline & Before PSM & After PSM \\
\hline Propensity Score & 0.82 & 0.02 \\
Male & -0.26 & -0.01 \\
Weight & -0.57 & -0.02 \\
Height & -0.47 & -0.01 \\
BMI & -0.33 & -0.01 \\
Age & -0.53 & -0.01 \\
Drinking more than & -0.28 & $<0.01$ \\
once a week & & \\
Smoking & & \\
$\quad$ smoking cessation & -0.07 & 0.01 \\
$\quad$ Smoking & -0.02 & $<0.01$ \\
\hline
\end{tabular}

\section{Figure 1 The ASDMs of the covariates before and after PSM}

\section{The prevalence of hypertension and logistic model results after PSM}

As shown in Table 5, hypertension prevalence in the treatment group was slightly higher than that of the control group with no statistical significance in differences after PSM $(6.2 \%$ vs $5.8 \%, \mathrm{P}=0.09)$. This pattern is repeated in the male stratum but with a statistically significant difference (treatment group vs control group: $9.0 \%$ v $6.2 \%$, $\mathrm{P}<0.01)$. The opposite pattern was found in the female stratum - the prevalence of hypertension of female employees in the treatment group was significantly lower than that of the control group (treatment group $v s$ control group: $2.9 \% v s 5.4 \%, \mathrm{P}<0.01$ ). Furthermore, hypertension prevalence of the treatment group in those strata with relatively older age (31-45 and 46-60 years), larger BMI (Overweight and Obesity) and 
unhealthy lifestyle (drinking, smoking and smoking cessation) was higher (to various degrees). However, it is significantly lower in those strata with younger age (19-30 years), smaller BMI (normal or underweight) and healthier lifestyle (no drinking and no smoking groups).

Table 5 Comparisons of the hypertension prevalence after PSM $\left(\mathrm{N}^{*}, \%\right)$

\begin{tabular}{llll}
\hline Factors & Treatment Group & Control group & $\boldsymbol{P}$ Value \\
\hline $\begin{array}{l}\text { Overall } \\
\text { Gender }\end{array}$ & $1,612(6.2)$ & $1,520(5.8)$ & 0.09 \\
$\quad$ Male & $1,261(9.0)$ & $859(6.2)$ & $<0.01$ \\
$\quad$ Female & $351(2.9)$ & $661(5.4)$ & $<0.01$ \\
Age & & & \\
$\quad$ 19-30 & $617(3.3)$ & $637(3.4)$ & 0.04 \\
$31-45$ & $781(12.4)$ & $692(11.2)$ & 0.68 \\
$46-60$ & $214(20.0)$ & $191(19.2)$ & 0.71 \\
BMI & & & \\
$\quad$ Normal or underweight & $42(0.8)$ & $15(0.2)$ & $<0.01$ \\
Overweight & $603(4.2)$ & $406(3.5)$ & $<0.01$ \\
$\quad$ Obesity & $967(15.0)$ & $1,099(14.6)$ & 0.53 \\
Drinking & & & \\
More than once a week & $707(11.4)$ & $390(6.2)$ & $<0.01$ \\
$\quad$ No drinking & $905(4.5)$ & $1,130(5.7)$ & $<0.01$ \\
Smoking & & & \\
No smoking & $668(3.8)$ & $961(5.5)$ & $<0.01$ \\
$\quad$ Smoking cessation & $112(18.6)$ & $85(13.8)$ & 0.02 \\
Smoking & $832(10.5)$ & $474(6.0)$ & $<0.01$ \\
\hline
\end{tabular}

*N was the number of subjects with hypertension.

Table 6 shows the results of unadjusted and adjusted logistic models performed with overall samples after PSM. The unadjusted logistic model results show that the treatment group is more vulnerable to hypertension than the control group $(\mathrm{OR}=1.20$, $95 \% \mathrm{CI}=1.11-1.29, \mathrm{P}=0.03)$. A similar result is found in the adjusted logistic model where BMI and age are incorporated as covariates, even though the OR value drops to 1.1 (95\% CI: 1.02-1.19, $\mathrm{P}=0.01)$. In terms of $\mathrm{BMI}$ and age in the adjusted model, statistically significant gradient effects on hypertension are found, which indicates that both in the treatment and control groups, those with larger BMI and older age are more likely to be hypertensive.

Table 6 Results of unadjusted and adjusted logistic models after PSM

\begin{tabular}{|c|c|c|c|c|c|}
\hline & \multicolumn{2}{|c|}{ Unadjusted Model } & \multicolumn{3}{|c|}{ Adjusted Model } \\
\hline & OR $95 \%$ CI & P value & OR & $95 \% \mathrm{CI}$ & P value \\
\hline \multicolumn{6}{|l|}{ Group } \\
\hline Treatment group & 1 & & 1 & & \\
\hline
\end{tabular}




\begin{tabular}{|c|c|c|c|c|c|}
\hline Control group & \multicolumn{2}{|c|}{$1.201 .11-1.290 .03$} & 1.10 & 1.02-1.19 & 0.01 \\
\hline \multicolumn{6}{|l|}{ BMI } \\
\hline Normal or underweight & - & - & 1 & & \\
\hline Overweight & - & - & 2.91 & $2.69-3.16$ & $<0.01$ \\
\hline Obesity & - & - & 5.66 & $4.98-6.44$ & $<0.01$ \\
\hline \multicolumn{6}{|l|}{ Age } \\
\hline 18-30 years & - & - & 1 & & \\
\hline $31-45$ years & - & - & 7.35 & $5.62-9.61$ & $<0.01$ \\
\hline $46-60$ years & - & - & 28.49 & $21.85-37.15$ & $<0.01$ \\
\hline
\end{tabular}

Table 7 and Table 8 respectively show the results of the gender sub-group analysis after PSM. Table 7 shows that, in the male group, the treatment group is more vulnerable to hypertension than the control group (unadjusted model: $\mathrm{OR}=1.51$, 95\% $\mathrm{CI}=1.38-1.65, \mathrm{P}<0.01$; adjusted model: $\mathrm{OR}=1.43,95 \% \mathrm{CI}=1.31-1.58, \mathrm{P}<0.01$ ). Conversely, Table 8 shows that, in the female group, the treatment group is less vulnerable to hypertension than the control group (unadjusted model: $\mathrm{OR}=0.52$, 95\% CI $=0.46-0.59, \mathrm{P}<0.01$; adjusted model: $\mathrm{OR}=0.64,95 \% \mathrm{CI}=0.56-0.73, \mathrm{P}<0.01$ ). Similar results are found in both male and female groups for BMI and age which are both significantly associated with hypertension. The ORs in these two variables increase as the levels become higher, which indicates the gradient effects on hypertension.

Table 7 Unadjusted and adjusted logistic models after PSM (male group)

\begin{tabular}{|c|c|c|c|c|c|c|}
\hline \multirow{2}{*}{ Factors } & \multicolumn{3}{|c|}{ Unadjusted Model* } & \multicolumn{3}{|c|}{ Adjusted Model** } \\
\hline & $\mathbf{O R}$ & $95 \% \mathrm{CI}$ & $P$ value & $\mathbf{O R}$ & $95 \% \mathrm{CI}$ & $P$ value \\
\hline \multicolumn{7}{|l|}{ Group } \\
\hline Treatment group & 1 & & & 1 & & \\
\hline Control group & 1.51 & 1.38-1.65 & $<0.01$ & 1.43 & 1.31-1.58 & $<0.01$ \\
\hline \multicolumn{7}{|l|}{ BMI } \\
\hline Normal or underweight & & - & - & & & \\
\hline Overweight & - & - & - & 2.48 & $2.25-2.74$ & $<0.01$ \\
\hline Obesity & - & - & - & 4.72 & $4.03-5.52$ & $<0.01$ \\
\hline \multicolumn{7}{|l|}{ Age } \\
\hline $18-30$ years & - & - & - & & & \\
\hline $31-45$ years & - & - & - & 7.62 & $5.48-10.59$ & $<0.01$ \\
\hline $46-60$ years & - & - & - & 26.15 & $\begin{array}{l}18.90- \\
36.18\end{array}$ & $<0.01$ \\
\hline
\end{tabular}

*: The covariate of the logistic model just included treatment/control group.

**: The covariate of the logistic model included treatment/control group, BMI and age.

Table 8 Unadjusted and adjusted logistic models after PSM (female group)

Factors $\quad$ Unadjusted Model $\quad$ Adjusted Model




\begin{tabular}{lllllll} 
& OR & $\mathbf{9 5 \%}$ CI & P value & OR & $\mathbf{9 5 \%}$ CI & P value \\
\hline $\begin{array}{l}\text { Group } \\
\quad \text { Treatment group }\end{array}$ & $\mathbf{1}$ & & & $\mathbf{1}$ & & \\
$\quad$ Control group & $\mathbf{0 . 5 2}$ & $\mathbf{0 . 4 6 - 0 . 5 9}<\mathbf{0 . 0 1}$ & $\mathbf{0 . 6 4}$ & $\mathbf{0 . 5 6 - 0 . 7 3}$ & $<\mathbf{0 . 0 1}$ \\
BMI & & & & & & \\
$\quad$ Normal or underweight & - & - & - & 1 & & \\
$\quad \begin{array}{l}\text { Overweight } \\
\text { Obesity }\end{array}$ & - & - & - & 3.15 & $2.73-3.63$ & $<\mathbf{0 . 0 1}$ \\
Age & - & - & - & 6.54 & $5.21-8.20$ & $<\mathbf{0 . 0 1}$ \\
$\quad$ 18-30 years & - & - & - & 1 & & \\
31-45 years & - & - & - & 8.07 & $5.07-12.82$ & $<\mathbf{0 . 0 1}$ \\
46-60 years & - & - & - & 33.08 & $20.87-$ & $<\mathbf{0 . 0 1}$ \\
\end{tabular}

*: The covariate of the model just included treatment/control group.

**: The covariates of the model included treatment/control group, BMI, and age.

\section{Discussion}

Changqing Oil Field is a large state-owned oil and gas company, with nearly 80,000 employees, headquartered in Shaanxi Province. Employees in oil and gas enterprises are usually exposed to more occupational health risk factors than the general workforce, which may lead to a higher risk of chronic disease, including hypertension. However, the average incomes of employees in this company are higher and medical care is much better, which can have certain protective effects on chronic diseases that may, in turn, offset the negative effects of exposure to occupational health risk factors. Furthermore, chronic diseases are affected not only by the above external factors but also by internal individual factors, including for example, demographic and health behavior factors. For these reasons, we argue that when evaluating the efficacy of health management measures, companny's internal health management departments and more generally health policy makers, should increase the comparability and reliability of results by balancing the influence of these factors, before comparing the prevalence of chronic diseases between their employees and the general population.

In the current study, employees in Changqing Oil Field are younger on average but have unhealthier lifestyles with higher BMI and a greater incidence of drinking and smoking. Also, for the company examined, there is a higher proportion of male employees. Related and as shown in previous studies, age, gender and lifestyle are strongly associated with hypertension. ${ }^{21}$ Therefore, if we directly compare the prevalence of hypertension without adjusting for the above confounding factors, this may potentially bias the results. The results of the direct comparison of the prevalence 
of hypertension showed that the direction of the difference in different strata between the two groups was not consistent. For example, in Changqing Oil Field, the hypertension prevalence of those strata who were female, aged 19-30, non-drinker and non-smoker were much lower. This imbalance in covariates between the two groups examined in this study, meant that it was necessary to balance the multiple confounding factors using an appropriate technique i.e. PSM.

After using the PSM method to balance the confounders measured in the two surveys (such as age, gender, BMI, smoking and drinking), the results for both the unadjusted and adjusted (by BMI, age and gender) models showed that Changqing employees were more vulnerable to hypertension than Shaanxi residents. One possible reason is that employees in oil and gas enterprises are more likely to be exposed to occupational hazards such as physical factors (dust, high temperature and pressure, noise and power frequency electromagnetic fields) and chemical factors (including H2S, aromatic hydrocarbons, alkanes, etc.). ${ }^{8-9}$ If the enterprise's protective facilities were defective, or the staff's self-protection consciousness was not strong, this may increase the risk of hypertension. A second possible explanation is that Changqing employees' workplaces are mostly located in remote areas, far from the city. The costs associated with transportation and time taken for workers to go to their health organization are therefore realtively high, which may result in reduced use of available medical and health services. Lastly, many oil and gas enterprises implement a 24-hour shift system for front-line jobs. Relevant studies have shown that shift work patterns can cause shift work sleep disorder (SWD) which can lead to biological rhythm disorders, such as changes in dietary behavior, insomnia, etc., thereby increasing the risk of hypertension. ${ }^{10}$

Another interesting finding is that after PSM, in the male subgroup, Changqing employees are more vulnerable to hypertension than Shaanxi residents, while the opposite is found in the female subgroup. This may be due to any of the following reasons. First, Changqing's male employees are mostly engaged in front-line and outdoor work. Their work intensity is greater and their working environment is worse. This is in contrast to female employees who are mostly in logistics departments and indoor positions. On the whole, female working and living conditions were relatively better. Second, female workers in Changqing Oil Field are, by definition, paid employees while most of the female residents in Shaanxi Province live in the countryside, where women are mostly unemployed and receive less health education. Therefore their willingness and ability to purchase medical services may be inferior to 
those of women working in the Changqing Oil Field.

An important strength of this study is that the data sources are from two largescale, high-quality surveys. Within the surveys, the data of Shaanxi residents came from Shaanxi's 2013 NHSS - a large-scale provincial representative survey. The participants of Changqing survey accounted for about $60 \%$ of all staff and covered all types of employees. Therefore, the two samples well represent their respective populations. Another strength of this study is that the two groups are in the same region. Therefore, apart from occupational characteristics, the two groups are similar, which in turn increases comparability. Furthermore, use of the PSM method to balance the distribution of age, gender, BMI, smoking and drinking within the two groups, improves the comparability of hypertension prevalence between the two groups. A limitation of this study is that Changqing employees were not randomly selected, which may lead to selection bias. However, after correcting for the main confounding factors of hypertension using PSM and the logistic model, we believe that potential biases have been effectively reduced. Another limitation is that although several confounding factors (such as age, gender, BMI, smoking and alcohol consumption) are corrected, some unobserved confounding factors were not adjusted. Hence, bias due to this potential omission has not been accounted for.

In conclusion, we find that Changqing employees were more vulnerable to hypertension than the general residents in Shaanxi Province. This study suggests that, with regards to policies concerning the prevention, treatment and control of hypertension, the department of health management in the company should formulate different policies for male and female workers respectively and should be intensely focused male and front-line workers.

\section{Strengths and limitations of this study}

- This study compares the hypertention prevalence of employees in oil and gas industries with general population of working age for the first time.

- Data is derived from large-scale, high-quality health surveys performed in the in the same geographical region.

- Results provide implication that the company's health management department should formulating different policies for male and female workers respectively and should be intensely focused male and front-line workers.

- Cross-sectional study design precludes the demonstration of a causal relationship between the vulnerability of hypertention and the characteristic of the employees.

- Unobserverd important confoundings cann't be adjusted by using PSM method.

\section{Acknowledgements}

The author sincerely thanks the editors and anonymous reviewers for their helpful suggestions and valuable comments. 


\section{Author's contributors}

Ying Liang undertook the data analysis in conjunction with Tianle Che and Zhijun Tan. All authors contributed to data interpretation. Ling Li and Hai Mi participated in the data collection of the Health Risk Factors Survey in Changqing Oil Field Filiale. Ying Liang, Kyriacos Kyriacou and Siming Liu drafted the manuscript and all authors contributed to its development.

\section{Author's information}

Email Adresses for the Co-authors:

Ying Liang: liangying1937@126.com

Tianle Che: wanzhuanmeitian@outlook.com

Kyriacos Kyriacou: Kyriacos.Kyriacou@brunel.ac.uk

Siming Liu: Siming.Liu@brunel.ac.uk

Haiyue Zhang: zhyue0317@163.com

Ling Li: 346269695@qq.com

Hai Mi: 610628583@qq.com

Shang Lei:shangleitg@126.com

Funding : This work has been funded by the National Natural Science Foundations (No. 81703315, No. 81773540 ) and Key R \& D plan of Shaanxi Province (2020SF143,2020 SF-288 ). The funder did not play any role in the study.

\section{Declarations}

Competing interests: The authors declare that they have no competing interests.

Patient consent for publication: Not required.

Ethics approval: The HRFS was approved by the Department of Health management of the Changqing Oil Field Filiale. The NHSS obtained ethical approval (license number: 2013 (65)) from the institutional review board of the Chinese National Bureau of Statistics. Both of the two surveys have pledged to protect the privacy of respondents and to facilitate the anonymous analysis of the data according. For these reasons, a written consent was not required. The informed consent statement was printed on the questionnaire to ask for the interviewees' permission.

Provenance and peer review: Not commissioned; externally peer reviewed.

Data availability statement: No additional data are available.

Acknowledgments: We would like to thank the officers of the surveyed for their assistance and, the interviewers for their excellent field work, and all respondents for their cooperation.

Availability of data and materials: Data are available upon reasonable request. 


\section{Reference}

[1] Olsen Michael H, Angell Sonia Y, Asma Samira, et al.. A call to action and a lifecourse strategy to address the global burden of raised blood pressure on current and future generations: the Lancet Commission on hypertension. Lancet (London, England), 2016, 388 (10060).

[2] The Lancet. Hypertension: an urgent need for global control and prevention. The Lancet,2014,383(9932).

[3] Jeffrey D Stanaway, Ashkan Afshin,Emmanuela Gakidou,et al. Global, regional, and national comparative risk assessment of 84 behavioural, environmental and occupational, and metabolic risks or clusters of risks for 195 countries and territories, 1990-2017: a systematic analysis for the Global Burden of Disease Study 2017. The Lancet, 2018, 392(10159).

[4] Li YC, Wang LM, Jiang Y, et al. Prevalence of hypertension among Chinese adults in 2010. Zhonghua Yu Fang Yi Xue Za Zhi. 2012;46:409-413.

[5] Jiapeng Lu,Yuan Lu, Xiaochen Wang, et al. Prevalence, awareness, treatment, and control of hypertension in China: data from 1.7 million adults in a population-based screening study (China PEACE Million Persons Project). The Lancet, 2017, 390(10112).

[6] Danaei G, Finucane MM, Lin JK, et al. National, regional, and global trends in systolic blood pressure since 1980: systematic analysis of health examination surveys and epidemiological studies with 786 country-years and 5.4 million participants. Lancet. 2011; 377: 568-577.

[7] William K. Bosu. The prevalence, awareness, and control of hypertension among workers in West Africa: a systematic review. Global Health Action. 2015, 8(1).

[8] Mannocci A,Pignalosa S,Nicosia V, et al. Cardiovascular Diseases Risk Factors in oil and gas workers: a ten years observational retrospective cohort. Ann Ig 2016; 28:122-132.

[9] Poorabdian S, Mirlohi AH, Habibi E, et al. Association between job strain (high demand-low control) and cardiovascular disease risk factors among petrochemical industry workers. International Journal of Occupational Medicine and Environmental Health. 2013; 26(4):555-562.

[10] Mannocci A, Pignalosa S, Saulle R, et al. Prevalence of major cardiovascular risk factors among oil and gas and energy company workers. Ann Ist Super Sanita. 2015; 51(2):148-53.

[11]Burton WN,Conti DJ,Chen CY, et al. The role of health risk factors and disease on 
worker productivity. Journal of Occupational and Environmental Medicine, 1999;41(10).

[12]Poorabdian S, Mirlohi AH, Habibi E, et al. Association between job strain (high demand-low control) and cardiovascular disease risk factors among petrochemical industry workers. International Journal of Occupational Medicine and Environmental Health. 2013;26(4):555-562.

[13]Kadafa AA. Environmental impacts of oil exploration and exploitation in the Niger Delta of Nigeria. Glo J Sci Front Res 2012; 12:19-28.

[14] Ite AE, Ibok UJ. Gas flaring and venting associated with petroleum exploration and production in the Nigeria's Niger Delta. Am J Environ Protec 2013; 1:70-77.

[15]Chuang KJ, Yan YH, Cheng TJ. Effect of air pollution on blood pressure, blood lipids, and blood sugar: a population-based approach. J Occup Environ Med 2010; 52:258-262.

[16]Chuang KJ, Yan YH, Chiu SY, et al. Long-term air pollution exposure and risk factors for cardiovascular diseases among the elderly in Taiwan. Occup Environ Med 2011; 68:64-68.

[17]Dong G-H, Qian ZM, Xaverius PK, et al. Association between long-term air pollution and increased blood pressure and hypertension in China. Hypertension $2013 ; 61: 578-584$.

[18] Coogan PF, White LF, Jerrett M, et al. Air pollution and incidence of hypertension and diabetes mellitus in black women living in Los Angeles. Circulation 2012; 125:767-772.

[19]Ying Li. Constructing the Social Security Management System with Oil Field Characteristics. China Petroleum Enterprise, 2015(10):94-95.(In Chinese)

[20]Renata Cifkova,Jan Pitha,Alena Krajcoviechova, et al. Is the impact of conventional risk factors the same in men and women? Plea for a more genderspecific approach. International Journal of Cardiology, 2019, 286.

[21]Gerry Veenstra. Race, gender, class, sexuality (RGCS) and hypertension. Social Science \& Medicine, 2013, 89.

[22] Clougherty Jane E,Eisen Ellen A,Slade Martin D, et al. Gender and sex differences in job status and hypertension. OEM Online, 2010, 68(1).

[23] Austin Peter C. An Introduction to Propensity Score Methods for Reducing the Effects of Confounding in Observational Studies. Multivariate Behavioral Research, 2011, 46(3).

[24]Zhijun Tan, Ying Liang, Fuyan Shi, et al. Research on the CHD risk assessment 
model for workforce population from the perspective of enterprise health management. Modern Preventive Medicine, 2017, 44(12):2192-2195+2210.

[25] Austin PC. Some methods of propensity-score matching had superior performance to others: results of an empirical investigation and Monte Carlo simulations. Biometrical Journal.2009.51:171-184. 
Figures

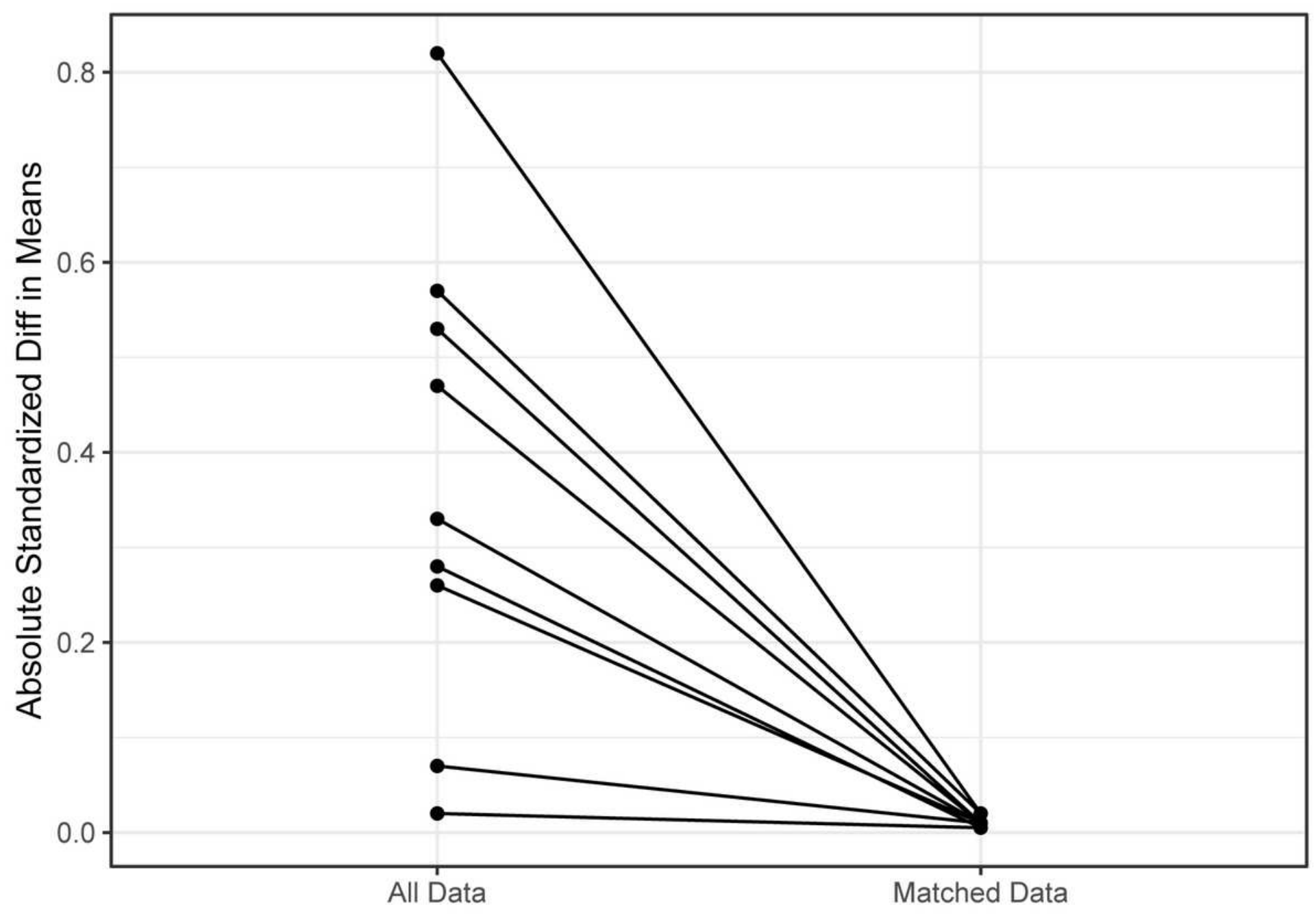

Figure 1

The ASDMs of the covariates before and after PSM 